\title{
Canadian guidelines on the management of colorectal peritoneal metastases
}

\author{
A. Brind'Amour MD, ${ }^{* \dagger}$ P. Dubé $M D_{1}^{* \ddagger}$ J.F. Tremblay MD, ${ }^{* \ddagger}$ M.L. Soucisse $M D^{* *}{ }^{* \ddagger}$ L. Mack MD, ${ }^{\S}$ \\ A. Bouchard-Fortier MD, ${ }^{\S}$ J.A. McCart $M D_{1} \| \#$ A. Govindarajan MD, ${ }^{\| \# ~ D . ~ B i s c h o f ~} M D_{,}^{\| \#}$ \\ E. Haase $M D,{ }^{* *}$ C. Giacomantonio $M D_{,}^{+\dagger}$ P. Hebbard $M D,{ }^{\neq \neq}$R. Younan $M D,{ }^{\neq \S \S}$ A MacNeill MD, IIII \\ C. Boulanger-Gobeil MD, ${ }^{\dagger}$ and L. Sidéris $\mathrm{MD}^{* \ddagger}$
}

\begin{abstract}
Modern management of colorectal cancer (CRC) with peritoneal metastasis (PM) is based on a combination of cytoreductive surgery (CRS), systemic chemotherapy, and hyperthermic intraperitoneal chemotherapy (HIPEC). Although the role of HIPEC has recently been questioned with respect to results from the PRODIGE 7 trial, the role and benefit of a complete CRS were confirmed, as observed with a 41-month gain in median survival in that study, and $15 \%$ of patients remaining disease-free at 5 years. Still, CRC with PM is associated with a poor prognosis, and good patient selection is essential. Many questions about the optimal management approach for such patients remain, but all patients with PM from CRC should be referred to, or discussed with, a PM surgical oncologist, because cure is possible. The objective of the present guideline is to offer a practical approach to the management of PM from CRC and to reflect on the new practice standards set by recent publications on the topic.
\end{abstract}

Key Words Peritoneal metastases, colorectal cancer, cytoreductive surgery, hyperthermic intraperitoneal chemotherapy, peritoneal carcinomatosis

Curr Oncol. 2020 December:27(6)e621-e631

www.current-oncology.com

\section{INTRODUCTION}

Colorectal cancer (CRC) is the 3rd most commonly diagnosed cancer and the 4 th leading cause of cancer-related mortality in the world ${ }^{1}$. At the time of diagnosis, $25 \%$ of patients have metastatic disease, and up to $10 \%$ have synchronous peritoneal metastases $(\mathrm{PMs})^{2,3}$. More than half the patients with recurrent disease will present with metachronous PMs, believed to be cancer cells disseminated during the index resection of the primary tumour. The global burden of CRC is expected to increase by $60 \%$ in the next 10 years worldwide ${ }^{1}$, and although the peritoneum is the only dissemination site in about $5 \%$ of cases, the incidence of patients with PMs is therefore also expected to increase.

In selected patients, management of PMs from CRC is based on a combination of cytoreductive surgery (CRS), systemic chemotherapy, and hyperthermic intraperitoneal chemotherapy (HIPEC). In the late 1980s, Sugarbaker first described the curative potential of that combination for patients with PMs from $\mathrm{CRC}^{4}$, which was confirmed by many studies in the years that followed ${ }^{5-12}$. However, recurrence rates remain high, and PM is still considered a negative presentation of CRC, associated with a poor prognosis ${ }^{13}$. For example, the PRODIGE 7 trial $^{14}$, a randomized trial evaluating the benefits of HIPEC after complete CRS of PMs from CRC, was presented at the American Society of Clinical Oncology meeting in June 2018 and reported a 15\% cure rate at 5 years (not yet published), the best results ever reported in a controlled study for such a cohort.

In 2015, in an effort to meet patient need and to ensure the highest standard of care possible, the Canadian HIPEC Collaborative Group published guidelines for the use of CRS and HIPEC in patients with PMs arising from $\mathrm{CRC}^{15}$. Since then, several important studies leading to changes in practice have been published or presented. Those studies also recently led expert groups from France ${ }^{16}$, Spain ${ }^{17}$, and the United States ${ }^{18}$ to publish new guidelines for the management of affected patients. Here, we offer an up-to-date and practical approach to the management of PMs from CRC and a reflection about the new practice standards in Canada.

\section{METHODS}

A search of PubMed was conducted to obtain an updated overview of the literature describing the current management of PMs arising from CRC. The key words used were 
“peritoneal carcinomatosis," "peritoneal metastases," "PC," "intraperitoneal chemotherapy," “HIPEC," “colorectal neoplasms" (or cancers), "colonic neoplasms" (or cancers), "rectal neoplasms" (or cancers), and "chemohyperthermia." Descriptive studies and clinical trials (phases II and III) published between 1990 and 2020 were retained. A first version of this manuscript was written after that exercise. A panel of surgical experts (AB, PD, JFT, MLS, LM, ABF, JAM, AG, DB, EH, CG, PH, RY, AM, CBG, LS) from each centre specialized in peritoneal surface oncology in Canada then met in teleconference in May 2020 to propose a consensus expert opinion on the management of PMs from CRC and to discuss the manuscript. A final version was thereafter sent to each author for critical review and final approval before publication.

\section{Patient Selection}

To be considered for surgical management of PMs, patients should be fit enough to undergo a high-risk procedure and be exempt from any major comorbidities ${ }^{19}$. Patients should have no signs of complete bowel obstruction, and their Eastern Cooperative Oncology Group performance status at the time of surgery should be less than $2^{20,21}$. Age greater than 65 years is no longer a contraindication, because many patients who are older but otherwise healthy have been operated on in recent years with uneventful postoperative courses $^{22-24}$. Although a recent meta-analysis by Gagnière et al. ${ }^{25}$ concluded that elderly patients experience increased postoperative mortality and morbidity, the authors insisted on considering frailty over age when selecting patients for CRS and HIPEC, because of the presence of major biases in the studies included in the analysis. Another important aspect to consider when evaluating older patients is that there are no available data about the impacts on postoperative quality of life and functional outcomes for that specific population ${ }^{26}$. In Canada, patients more than 65 years of age can be considered for CRS, but those more than 75 years are rarely considered. With respect to body mass index, a value above 35 is still a relative contraindication ${ }^{27,28}$, because patients with extreme obesity are subject to an increased rate of incomplete cytoreduction and shorter survival ${ }^{29}$. That being said, fitness for operation and Eastern Cooperative Oncology Group status are factors more important to consider than body mass index alone. Finally, patients must be motivated and understand the extent, risks, and potential benefits of the procedure.

\section{Preoperative Assessment}

These elements should be included when patients are referred to a PM surgical oncologist:

Complete history and physical examination, including previous chemotherapy and radiotherapy treatments Most recent blood tests, including tumour markers (carcinoembryonic antigen, cancer antigen 125, and carbohydrate antigen 19-9)

Most recent colonoscopy report

Previous operative procedures, especially if the patient underwent an exploratory surgery (such as a diagnostic laparoscopy) describing PM volume and distribution
Most recent imaging, including computed tomography imaging of chest, abdomen, and pelvis ${ }^{30,31}$, or integrated positron-emission tomography-computed tomography imaging ${ }^{32}$, or both

The addition of magnetic resonance imaging with diffusion-weighted imaging could be considered, given suggestions in the recent literature that it might be superior to computed tomography imaging in predicting operability ${ }^{33,34}$. It could also be useful in cases of unclear liver imaging.

Pathology review at an expert centre of any tissue biopsy performed before referral (should include differentiation grade, presence of signet ring cells, microsatellite instability, and $R A S$ and $B R A F$ status, when available ${ }^{35}$ )

In case of metachronous disease, pathology review of the previously resected primary should also be included.

\section{Acute Disease Presentation}

Acute presentation of PM in the presence of intestinal obstruction or perforation (or both) can represent a difficult challenge in terms of surgical management, because the surgeon has to act to resolve the patient's life-threatening condition without hindering further potentially curative treatments, including CRS and HIPEC. The operative goal in this type of scenario is to do only what is necessary to resolve the emergency, which can involve diverting stomas for obstruction and bowel resection for perforated tumours ${ }^{17}$ (Figure 1).

No attempt for CRS should be made in the emergency setting, even if the volume of PMs is limited, because such attempts are not associated with favourable oncologic outcomes $^{36}$ and can increase the difficulty of definitive surgery. However, an effort should be made to perform biopsies of PMs and to document their distribution in the peritoneal cavity. In the case of unfamiliarity with the peritoneal carcinomatosis index (PCI), a simple description of $\mathrm{PM}$ presence in each region of the abdomen can suffice ${ }^{37}$; extensive dissection to provide a more precise description should be avoided. Intraoperative photographs, especially when the procedure is laparoscopic, are very informative. General surgeons should not hesitate to contact a PM surgical oncologist when facing management difficulties in the emergency setting.

\section{Surgical Exploration and Excision}

Mechanical and oral antibiotic bowel preparation are given the day before surgery. Intravenous antibiotics are given at induction, and deep venous thrombosis prophylaxis is applied as in any other major abdominal surgery. To avoid compartment syndrome, which could occur after a lengthy procedure in which a perineal dissection is very rarely necessary, patients are positioned in the extended lithotomy position, with the legs set straight and spread ${ }^{38}$. Modified lithotomy position can be used as an alternative. An epidural catheter is installed before general anesthesia, together with arterial and central venous lines for most patients. Nasogastric and urinary catheters are routinely placed. Patients are prepped from the nipple line to the proximal thigh; female patients also receive vaginal prep. 


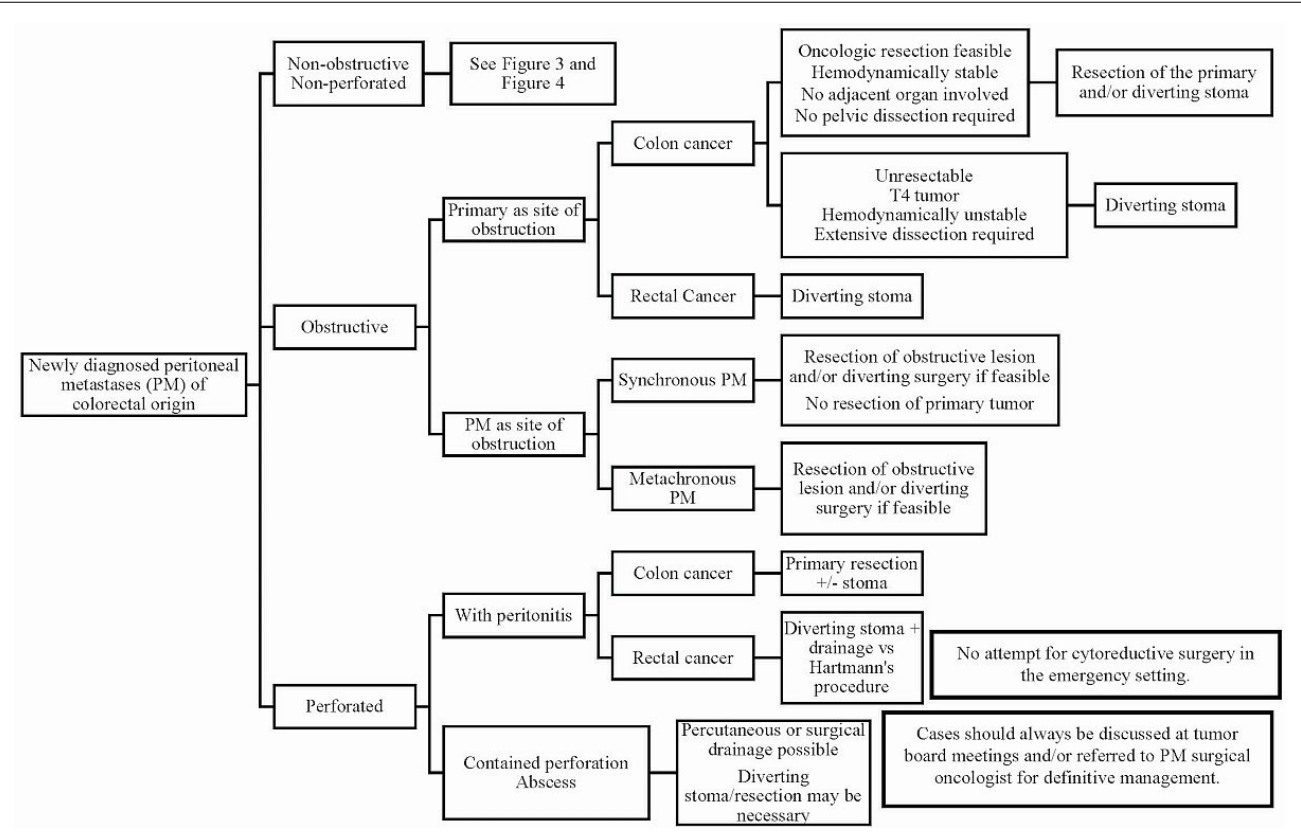

FIGURE 1 Algorithm for acute presentation of peritoneal metastases from colorectal cancer.

Cytoreductive surgery should be performed using a xyphopubic midline laparotomy ${ }^{16}$, which allows for complete visualization of the entire peritoneal cavity. Once the incision is made and the peritoneal cavity is accessible, a complete and thorough adhesiolysis is performed to evaluate the exact extent of disease by $\mathrm{PCI}^{39}$ and to allow for intraperitoneal chemotherapy diffusion after CRS. Frozen sections can be obtained at this point to confirm the diagnosis and to assess whether the disease biology has changed over time, which helps with intraoperative decision-making.

Although international experts have reached no definitive consensus concerning the ideal PCI cut-off at which to perform CRS for PMs of colorectal origin, the most widely accepted value is 20 or less for well-differentiated or moderately differentiated disease ${ }^{40-43}$, because a PCI greater than 20 is associated with a very poor prognosis ${ }^{44}$. For patients with poorly differentiated or signet ring adenocarcinoma, a PCI greater than 10 is a relative contraindication to surgery ${ }^{45-47}$. A complete CRS with no residual disease (completeness score: 0 ) should be the objective of cytoreduction for PMs of colorectal origin ${ }^{48}$ and an omentectomy should always be included. No CRS should be attempted if the disease is thought to be impossible to clear completely. Selective peritonectomy procedures should be performed as described by Sugarbaker ${ }^{49}$. Particular attention is given to the extent of disease involving the small bowel, also known as "small-bowel PCI," because recent literature has described it as an independent prognostic factor ${ }^{46,50}$. Although no cut-off has been determined specifically for small-bowel PCI, diffuse involvement of the small bowel is considered a contraindication to CRS. Other contraindications include the need for a Whipple procedure, definitive end stoma with concomitant ileal bladder (pelvic exenteration), major hepatectomy, and a bowel resection causing short-bowel syndrome ${ }^{15}$.
Protective stomas might be indicated in some cases with high-risk features for anastomotic leak (for example, multiple distal anastomoses, extensive CRS, or very low anterior resection) ${ }^{51}$, especially when pelvic radiation has been used in the past. However, we do not advocate for the routine use of protective stomas and tend to avoid their creation if possible, because their reversal is often difficult and can be associated with significant morbidity ${ }^{52,53}$. In contrast, the risk of anastomotic leakage as of today's standards for rectal anastomoses is deemed acceptable ${ }^{54}$. When consent for the operation is being sought, patients should always be informed of the risk of permanent stoma related to the CRS.

\section{HIPEC}

Delivery of HIPEC can be achieved using an open or closed technique; both techniques are safe and offer the same oncologic results ${ }^{55}$. Oxaliplatin and mitomycin $\mathrm{C}$ are the drugs most commonly used, but the use of other agents, such as doxorubicin, has also been described ${ }^{56,57}$. The preferred oxaliplatin regimen is $460 \mathrm{mg} / \mathrm{m}^{2}$ perfused for 30 minutes at $42^{\circ} \mathrm{C}$, together with systemic 5-fluorouracil $400 \mathrm{mg} / \mathrm{m}^{2}$ and leucovorin $20 \mathrm{mg} / \mathrm{m}^{2}$, administered 30-60 minutes before intraperitoneal chemotherapy ${ }^{58}$. The usual mitomycin C regimen is $40 \mathrm{mg}$ for $60-90$ minutes at $42^{\circ} \mathrm{C}$. Because many recent studies failed to demonstrate any survival difference between mitomycin $C$ and oxaliplatin ${ }^{59-62}$, both regimens are used in Canada according to local practice and experience. However, if the patient has shown recurrence or resistance to systemic oxaliplatin, mitomycin C should be favoured.

In an attempt to reduce disease recurrence and improve survival, use of early postoperative intraperitoneal chemotherapy (EPIC) in addition to CRS and HIPEC has, in recent years, been described for patients with PMs from CRC. Unfortunately, the earlier studies on the subject 
demonstrated increased postoperative morbidity and uncertain oncologic benefits ${ }^{63,64}$. Although more recent studies in patients with appendiceal tumours showed promising postoperative and long-term results ${ }^{65-67}$, we do not recommend the addition of EPIC for patients with CRC and PMs treated with CRS and HIPEC. The ICARus trial, an ongoing multicentre randomized controlled trial evaluating the effectiveness of EPIC after CRS and HIPEC for patients with PMs from appendiceal carcinoma and CRC (NCT01815359 at https://ClinicalTrials.gov/), should provide more information about the role of EPIC for such patients. Primary completion date is set for 2021.

The addition of HIPEC after CRS has been an active area of debate in the recent literature ${ }^{68-71}$ since the initial presentation of the PRODIGE 7 trial $^{14}$ in 2018. That randomized trial compared two cohorts of patients who underwent CRS for PMs from CRC, one adding HIPEC with high-dose oxaliplatin $\left(460 \mathrm{mg} / \mathrm{m}^{2}\right)$ at $43^{\circ} \mathrm{C}$ to CRS and the other involving CRS alone. The results showed no difference in 5-year overall survival [OS (Figure 2)] or disease-free survival (DFS) between the groups, apart from a subgroup analysis of patients with an intermediate PCI between 11 and 15 . The 5 -year os was $39.4 \%$ compared with $36.7 \%$ (hazard ratio: $1.00 ; 95 \%$ confidence interval: 0.73 to 1.37 ), and the 5 -year DFS was $14.8 \%$ compared with $13.1 \%$ (hazard ratio: 0.908 ; 95\% confidence interval: 0.69 to 1.19 ). Median survival was 41 months in the study, which is the best oncologic outcome ever reported in a controlled study for such a cohort. However, the authors found significantly increased postoperative morbidity in the HIPEC group (Table I). That observation led to practice changes by experts around the world ${ }^{16}$. Notably, patients in PRODIGE 7 were heavily treated in the neoadjuvant setting with oxaliplatin. With the trial being negative, some experts questioned whether oxaliplatin should be the HIPEC drug of choice in patients who have previously received multiple cycles of the drug and potentially developed resistant tumour clones ${ }^{59}$.

More research is necessary to understand the ideal agent, dose, duration, and heat exposure for HIPEC and to define the procedure's exact role in the treatment of colorectal PMs. Until then, adjustments to our practice were made in response to the recent literature. Some centres decided to stop using oxaliplatin for HIPEC because of PRODIGE 7's results; others still use oxaliplatin as the agent of choice, but now strongly consider performing CRS only for patients with a PCI less than 10, especially in cases of low-grade tumours, metachronous disease, or a patient with significant comorbidities.

A recent systematic review by Auer et al. ${ }^{72}$ concluded that, given only two randomized controlled trials on the subject, one being unpublished, HIPEC should not be used for the treatment of colorectal PMs outside a clinical trial. Although we acknowledge the low quantity of level 1 evidence supporting the use of HIPEC for affected patients, the difficulty in conducting such trials in this specific population, given the heterogeneity of disease presentation and the strict selection criteria for surgery, including the absence of synchronous extraperitoneal metastases, should be considered, as should expert opinion in the field ${ }^{18}$. Our experience of using HIPEC for the treatment of CRC metastases has been positive. For example, the group from Maisonneuve-Rosemont Hospital recently published a retrospective series of patients with PMs from CRC who underwent CRS and HIPEC during 2004-2015 ${ }^{73}$. The 91 patients who underwent CRS and oxaliplatin HIPEC had an os rate of $75 \%$ at 3 years and $55 \%$ at 5 years, with a median os duration of 63 months, and a DFS rate of $50 \%$ at 3 years and $25 \%$ at 5 years, with a median DFs duration of 36 months, demonstrating better oncologic outcomes than the current survival duration of 2 years with systemic chemotherapy alone ${ }^{72}$.

Although the way in which we administer HIPEC is now being reconsidered, especially since the presentation of the PRODIGE 7 trial, the role and benefit of CRs for patients with isolated PMs from CRC has been confirmed, achieving a much higher median survival than expected-more than 40 months in a controlled trial. An increased effort should be made to perform a complete CRs for patients with such a disease presentation, because cure is possible.

\section{Synchronous Isolated PMs}

Figure 3 presents our management guidelines for patients presenting with synchronous isolated PMs and CRC. The attitude toward perioperative systemic treatments has been controversial in the literature. A recent systematic

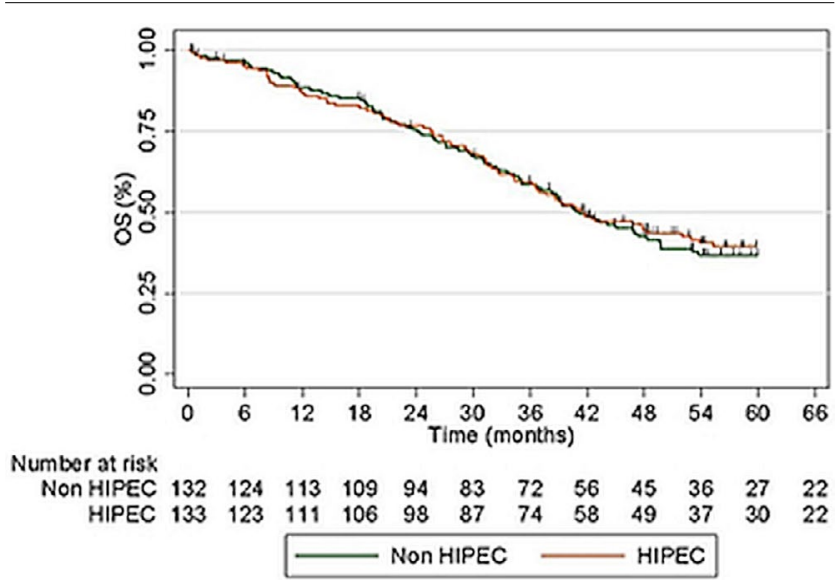

FIGURE 2 Overall survival in the PRODIGE 7 trial. HIPEC = hyperthermic intraperitoneal chemotherapy.

TABLE I Postoperative complications in the PRODIGE 7 trial

\begin{tabular}{|c|c|c|c|c|c|}
\hline \multirow{2}{*}{$\begin{array}{c}\text { Complication } \\
\text { type }\end{array}$} & \multicolumn{2}{|c|}{ HIPEC arm } & \multicolumn{2}{|c|}{ Non-HIPEC arm } & \multirow{2}{*}{$\begin{array}{c}p \\
\text { Value }\end{array}$} \\
\hline & (n) & $(\%)$ & (n) & $(\%)$ & \\
\hline \multicolumn{6}{|l|}{ All } \\
\hline All grades & 87 & 65.4 & 73 & 55.3 & 0.092 \\
\hline Grades 3-5 & 54 & 40.6 & 41 & 31.1 & 0.105 \\
\hline \multicolumn{6}{|l|}{ Intra-abdominal } \\
\hline All grades & 46 & 35.0 & 39 & 29.6 & 0.379 \\
\hline Grades 3-5 & 35 & 26.3 & 23 & 17.4 & 0.080 \\
\hline \multicolumn{6}{|l|}{ Extra-abdominal } \\
\hline All grades & 69 & 51.9 & 54 & 40.9 & 0.073 \\
\hline Grades 3-5 & 35 & 26.3 & 28 & 21.2 & 0.329 \\
\hline
\end{tabular}

HIPEC = hyperthermic intraperitoneal chemotherapy. 
review by Waite and Youssef ${ }^{74}$ found no evidence to support neoadjuvant systemic treatments, and limited evidence concerning the oncologic impact of adjuvant systemic therapies, in the absence of any randomized controlled trials conducted in that setting. Some groups advocate treating patients with upfront CRS ${ }^{75}$; others have had good results with neoadjuvant treatments ${ }^{76,77}$. Empirically, we usually give neoadjuvant systemic treatments to our patients, because we feel that they serve as good prognostic and predictive factors ${ }^{78}$, allowing us to test the tumour's biology and response to treatment ${ }^{79}$ and, in some cases, to help with patient scheduling. Furthermore, neoadjuvant systemic treatment permits patients who develop postoperative complications to be able to receive adjuvant treatments. Regimens such as FOLFOX (5-fluorouracilleucovorin-oxaliplatin), CAPOX (capecitabine-oxaliplatin), or FOLFIRI (5-fluorouracil-leucovorin-irinotecan) are usually used ${ }^{80-84}$. With respect to the addition of bevacizumab to those regimens, some series have reported that such an addition increases postoperative complications ${ }^{85}$, but the drug's role in the treatment of metastatic CRC is well established ${ }^{86}$, and its use has been demonstrated in previous studies to be safe and effective in patients with $\mathrm{PMs}^{78}$. We usually add bevacizumab to the neoadjuvant regimen for our patients, but stop it at least 6 weeks before any planned surgery. For patients with $R A S$ wild-type left-sided CRC, the addition of an anti-epidermal growth factor receptor type 1 agent (panitumumab) instead of bevacizumab could be considered $^{87,88}$ — with caution, because recent results in the neoadjuvant setting have been less encouraging than those in palliation ${ }^{89}$.
When fortuitously discovering PMs during elective colectomy, referring surgeons should consider aborting the resection of the primary (if the patient is asymptomatic), performing tissue biopsies, and documenting the disease's volume and distribution ${ }^{16}$. Such cases should always be discussed at a tumour board meeting or with a PM surgical oncologist (or both) for definitive management. In rare cases in which the PM burden is minimal and completely resectable without significantly extending the length or complexity of the surgery (for example, together with the primary's specimen or on the abdominal wall surface), an elective colectomy can be considered, but consultation with a PM surgical oncologist to help with the decision is strongly recommended.

\section{Metachronous Isolated PMs}

Figure 4 presents our management guidelines for patients presenting metachronous isolated PMs of colorectal origin. Metachronous disease is usually considered less aggressive than synchronous disease, because patients can develop PMs many years after their index colectomy. Such occurrences could possibly be related to differential disease biology, among other factors ${ }^{90}$. Management of such patients is different, because the primary has already been excised, and many patients have already received adjuvant chemotherapy (depending on the initial tumour's stage). Because disease recurrence is common ${ }^{91,92}$, patient selection is paramount. Timing of recurrence from index surgery, previous chemotherapy regimens, existence of previous or actual extraperitoneal disease, PCI, and tumour biology are important factors to consider.

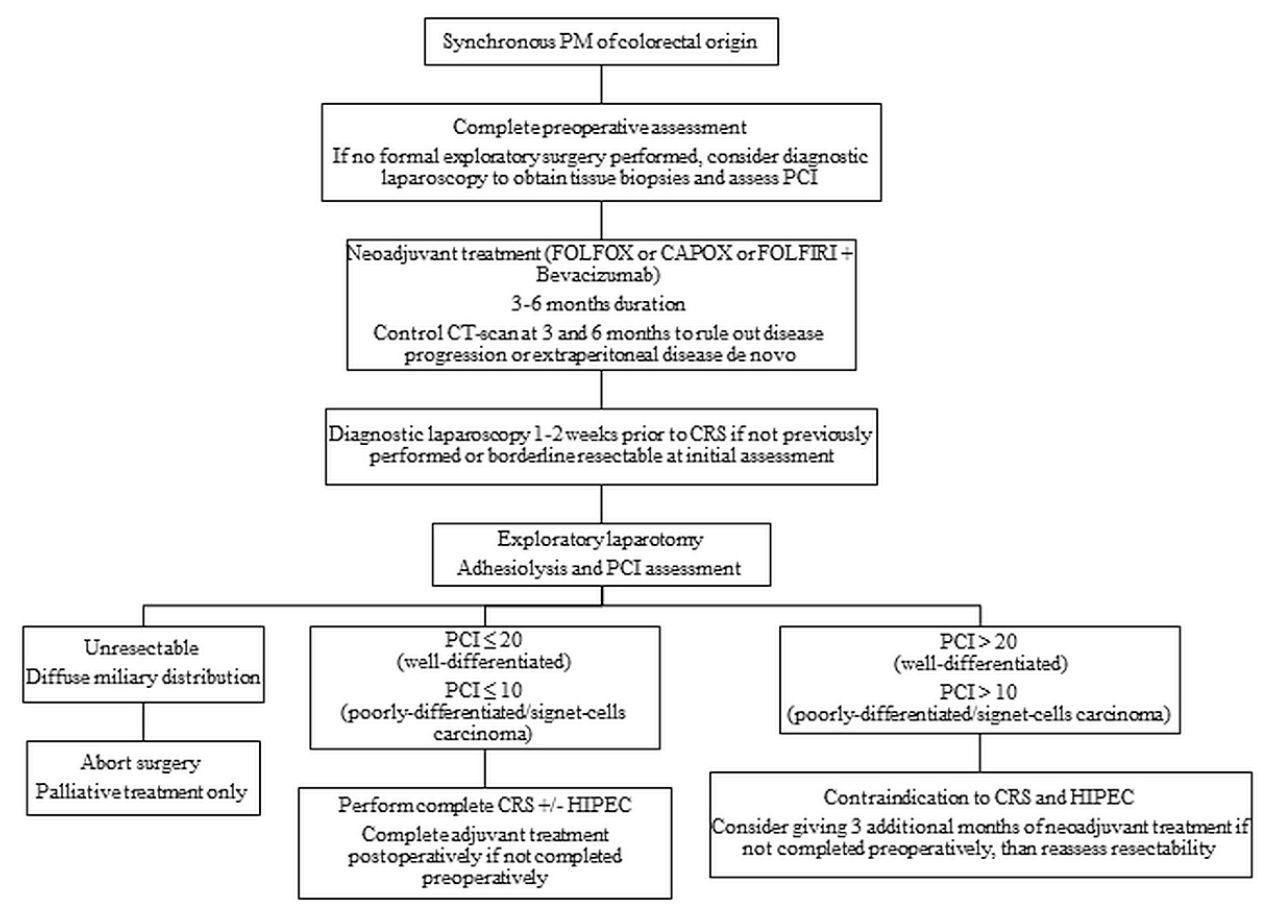

FIGURE 3 Standard management of synchronous isolated peritoneal metastasis (PM). PCI = peritoneal carcinomatosis index; FOLFOX = 5-fluorouracilleucovorin-oxaliplatin; CAPOX = capecitabine-oxaliplatin; FOLFIRI = 5-fluorouracil-leucovorin-irinotecan; $\mathrm{CT}=$ computed tomography; CRS = cytoreductive surgery; HIPEC = hyperthermic intraperitoneal chemotherapy. 


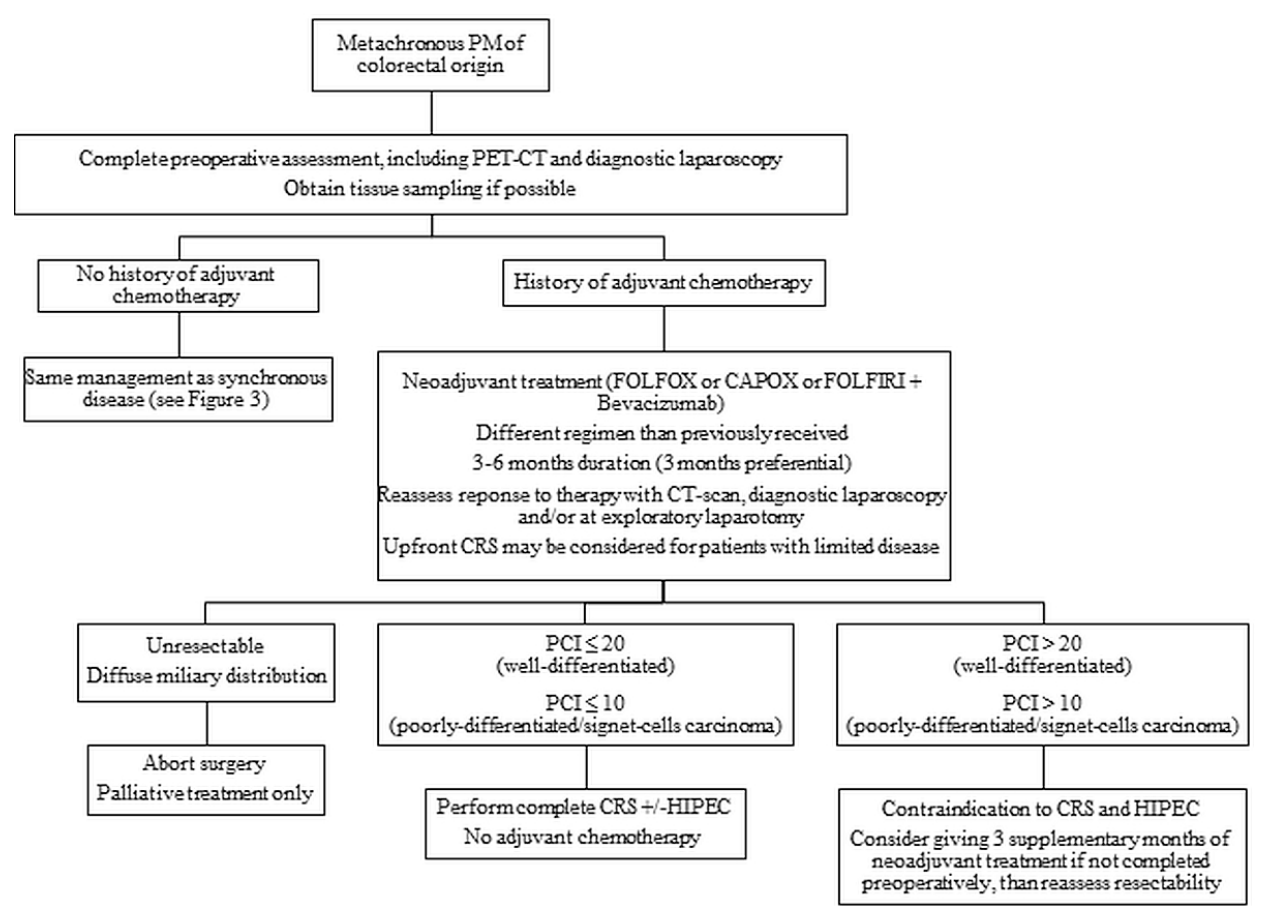

FIGURE 4 Standard management of metachronous isolated peritoneal metastasis (PM). PET-CT = integrated positron-emission tomography-computed tomography; FOLFOX = 5-fluorouracil-leucovorin-oxaliplatin; CAPOX = capecitabine-oxaliplatin; FOLFIRI = 5-fluorouracil-leucovorin-irinotecan; $\mathrm{CRS}=$ cytoreductive surgery; $\mathrm{PCI}=$ peritoneal carcinomatosis index; HIPEC = hyperthermic intraperitoneal chemotherapy.

The management of PM recurrence after CRS and HIPEC will not be discussed here, but the feasibility and oncologic benefit of repeat CRS and HIPEC has been demonstrated in the past ${ }^{93}$, and carefully selected patients are amenable to a second peritoneal therapy ${ }^{94}$.

\section{PM and Extraperitoneal Metastasis}

Synchronous extraperitoneal metastasis in the presence of PM is associated with poorer prognosis and considered a contraindication to CRS and HIPEC, because the presence of such metastasis generally demonstrates systemic disease for which a targeted abdominal surgery cannot be curative. Although most patients with extraperitoneal metastasis will not benefit from CRS and HIPEC ${ }^{95}$, recent literature has demonstrated that selected patients with PM and synchronous liver metastases (LMs) can benefit from combined CRS, HIPEC, and liver resection or ablative therapy ${ }^{96-98}$, because that approach can be associated with improved survival without increased morbidity and is more effective that modern systemic chemotherapy ${ }^{99}$. Currently, no guidelines about the optimal management of such patients are available, and the literature addressing the topic is very heterogeneous. When looking more specifically at the treatment of patients with synchronous PMs and LMs, various strategies have been proposed ${ }^{100}$. Although it was previously suggested that only patients with no more than 3 LMs could be considered for a curative approach ${ }^{15,101}$, that limit is no longer an exclusion criterion, provided that all metastases can be completely managed $^{102}$. Because more research will be necessary to determine which patients can benefit from aggressive treatment, a very selective "case-by-case" approach is recommended. Decision-making elements should include response to chemotherapy, onset presentation of both PMs and LMs with respect to the primary, number and distribution of LMs, PCI, and tumour biology.

The presence of enlarged retroperitoneal lymph nodes on imaging should not be considered an absolute contraindication to CRS and HIPEC. Retroperitoneal lymph nodes are extra-regional nodes, and their enlargement on preoperative imaging is therefore considered to demonstrate systemic spread of the disease. However, in a multicentric study, van der Werf et al. ${ }^{103}$ recently demonstrated that, compared with patients having normal retroperitoneal lymph nodes on imaging, patients with enlargement did not experience decreased DFS or Os. Although enlargement remains a relative contraindication, carefully selected patients with enlarged retroperitoneal lymph nodes on preoperative imaging could be considered for CRS with HIPEC, because the enlargement is not always cancer-related. Onset and extension of retroperitoneal disease, presence of other extraperitoneal metastases, response to systemic chemotherapy, and tumour biology are all factors that should be considered. However, if an intraoperative tissue biopsy is conclusive for retroperitoneal metastases, CRS and HIPEC should not be performed.

\section{High-Risk Features and "Prophylactic" HIPEC}

Figure 5 presents our revised management guidelines regarding second-look surgery and prophylactic HIPEC. The role of HIPEC as a prophylactic strategy to prevent the development of PMs in patients at high risk for peritoneal recurrence is controversial. A retrospective study by Elias et al. ${ }^{104}$ in 2011 demonstrated an oncologic benefit to 


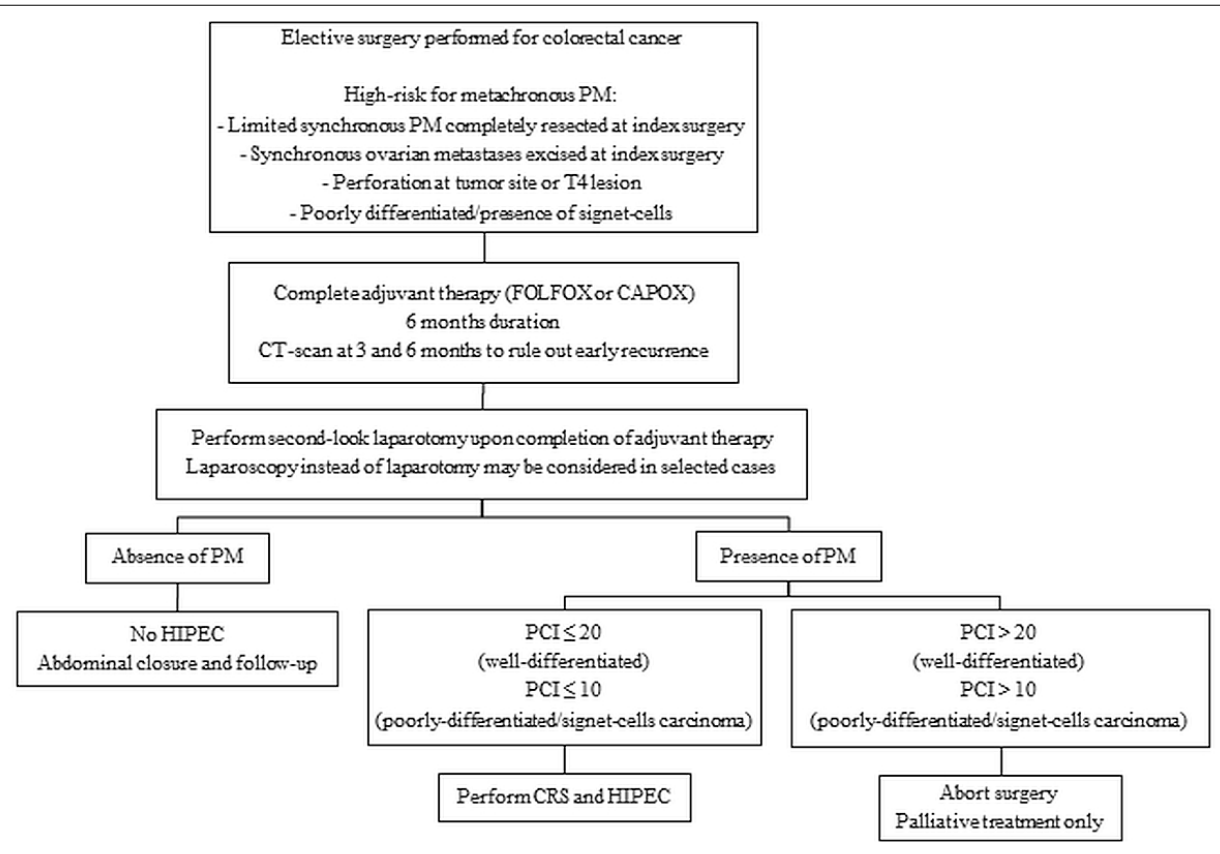

FIGURE 5 Standard management of patients with high-risk features for peritoneal recurrence. $\mathrm{PM}=$ peritoneal metastasis; FOLFOX = 5-fluorouracil-leucovorin-oxaliplatin; CAPOX = capecitabine-oxaliplatin; CT = computed tomography; HIPEC = hyperthermic intraperitoneal chemotherapy; $\mathrm{PCl}=$ peritoneal carcinomatosis index; $\mathrm{CRS}=$ cytoreductive surgery.

performance of a systematic second-look surgery and HIPEC for patients with high-risk features at index surgery-for example, synchronous minimal PMs completely excised at surgery, synchronous ovarian metastases, and perforation at the tumour site-with a 5 -year DFS of $44 \%$ and a 5 -year os of $90 \%$. Based on the results of that study, our approach was, until recently, to offer systematic second-look surgery and HIPEC to patients presenting such features or poor tumour biology (poorly differentiated disease or the presence of signet ring cells), another important risk factor for peritoneal recurrence ${ }^{17}$. Two important phase III randomized trials have since been conducted to examine the safety and oncologic benefit of the approach. The PROPHYLOCHIP trial ${ }^{105}$ considered postoperative surveillance only compared with systematic second-look laparotomy plus oxaliplatin-based HIPEC for patients with high-risk features (as already mentioned). The preliminary results showed no added morbidity, but no DFS or os benefit either after 3 years of follow-up, with a DFS of $44 \%$ compared with $51 \%$ ( $p=0.75$ ) and an os of $80 \%$ compared with $79 \%$. The COLOPEC trial ${ }^{106}$ considered adjuvant therapy alone compared with adjuvant therapy plus HIPEC for patients with T4 or perforated tumours. The preliminary results at 18 months showed no benefit for the addition of HIPEC, with a PM DFS of $81 \%$ compared with $76 \%$ (hazard ratio: 0.86; $95 \%$ confidence interval: 0.51 to 1.54 ).

While the final results of those studies are awaited, prophylactic HIPEC should no longer be systematically performed if no PM is found at second-look surgery. Second-look laparotomy or laparoscopy should still be considered, because the recurrence rate for such patients is high. As for the role of laparoscopy as a second-look method, the COLOPEC 2 trial ${ }^{107}$, a phase III multicentric randomized trial, is currently investigating the role of laparoscopy in second- and third-look surgery for patients with T4 tumours. It is the first study to specifically study the role of laparoscopy in that setting. Primary completion date is set for 2021.

\section{SUMMARY}

Modern management of PMs from CRC is based on a combination of radical surgery and perioperative systemic chemotherapy. Although the role of HIPEC has recently been questioned with respect to the results emerging from PRODIGE 7, the role and benefit of a complete CRS have been confirmed, as observed with the 41-month gain in os in that study, with $15 \%$ of patients cured at 5 years. Still, CRC with PMs is associated with a poor prognosis, and good patient selection is crucial. Finally, although many questions remain with respect to the optimal management approach for affected patients, all patients with PMs from CRC should be referred to, or discussed with, a PM surgical oncologist, because cure is possible. Further studies involving all Canadian HIPEC centres will be conducted in the near future in an effort to improve the quality of care given to our patients.

The objective of this guideline was to offer a practical approach to the management of PMs from CRC, to streamline the care of patients, and to reflect on the new practice standards set by recent publications on the subject. Given that more research will be necessary to define the exact role of HIPEC in the curative setting, it will be interesting to follow the emergence of pressurized intraperitoneal aerosol chemotherapy, a new drug delivery system for patients not amenable to CRS and HIPEC. Although the technique is relatively new, its feasibility and safety have already been demonstrated in the palliative setting for unresectable 
disease $^{108}$, and some groups have been studying its role as a potential bridge to a curative approach ${ }^{109}$.

\section{CONFLICT OF INTEREST DISCLOSURES}

We have read and understood Current Oncology's policy on disclosing conflicts of interest, and we declare that we have none.

\section{AUTHOR AFFILIATIONS}

*Department of Surgery, Hôpital Maisonneuve-Rosemont, Montreal, 'Department of Surgery, CHU de Québec-Université Laval, Quebec City, and ${ }^{\ddagger}$ Department of Surgery, University of Montreal, Montreal, QC; ${ }^{\S}$ Department of Surgery, University of Calgary, Calgary, AB; "Department of Surgery, Mount Sinai Hospital, and ${ }^{\#}$ Department of Surgery, University of Toronto, Toronto, $\mathrm{ON}$; **Department of Surgery, University of Alberta, Edmonton, $\mathrm{AB} ;{ }^{\dagger \dagger}$ Department of Surgery, Dalhousie University, Halifax, NS; 㧊Department of Surgery, University of Manitoba, Winnipeg, MB; ${ }^{\$ \S}$ Department of Surgery, Centre Hospitalier de l'Université de Montréal, Montreal, QC; IIIIDepartment of Surgery, University of British Columbia, Vancouver, BC.

\section{REFERENCES}

1. Arnold M, Sierra MS, Laversanne M, Soerjomataram I, Jemal A, Bray F. Global patterns and trends in colorectal cancer incidence and mortality. Gut 2017;66:683-91.

2. Massalou D, Benizri E, Chevallier A, et al. Peritoneal carcinomatosis of colorectal cancer: novel clinical and molecular outcomes. Am J Surg 2017;213:377-87.

3. Sugarbaker PH. Colorectal cancer: prevention and management of metastatic disease. Biomed Res Int 2014;2014:782890.

4. Sugarbaker PH. Surgical treatment of peritoneal carcinomatosis: 1988 Du Pont lecture. Can J Surg 1989;32:164-70.

5. Elias D, Goere D. Peritoneal carcinomatosis of colorectal origin: recent advances and future evolution toward a curative treatment. Recent Results Cancer Res 2007;169:115-22.

6. Elias D, Raynard B, Farkhondeh F, et al. Peritoneal carcinomatosis of colorectal origin. Gastroenterol Clin Biol 2006; 30:1200-4

7. Verwaal,VJ, Bruin S, Boot $H$, van Slooten $G$, van Tinteren $H$. 8-Year follow-up of randomized trial: cytoreduction and hyperthermic intraperitoneal chemotherapy versus systemic chemotherapy in patients with peritoneal carcinomatosis of colorectal cancer. Ann Surg Oncol 2008;15:2426-32.

8. Roviello F, Caruso S, Marrelli D, et al. Treatment of peritoneal carcinomatosis with cytoreductive surgery and hyperthermic intraperitoneal chemotherapy: state of the art and future developments. Surg Oncol 2011;20:e38-54.

9. Sayag-Beaujard AC, Francois Y, Glehen O, et al. Treatment of peritoneal carcinomatosis in patients with digestive cancers with combination of intraperitoneal hyperthermia and $\mathrm{mi}-$ tomycin C. Bull Cancer 2004;91:E113-32.

10. Elias D, Delperro JR, Sideris L, et al. Treatment of peritoneal carcinomatosis from colorectal cancer: impact of complete cytoreductive surgery and difficulties in conducting randomized trials. Ann Surg Oncol 2004;11:518-21.

11. Sugarbaker PH. Treatment of peritoneal carcinomatosis from colon or appendiceal cancer with induction intraperitoneal chemotherapy. Cancer Treat Res 1996;82:317-25.

12. Glehen O, Gilly FN, Boutitie F, et al. on behalf of the French Surgical Association. Toward curative treatment of peritoneal carcinomatosis from nonovarian origin by cytoreductive surgery combined with perioperative intraperitoneal chemotherapy: a multi-institutional study of 1,290 patients. Cancer 2010;116:5608-18.

13. Franko J, Shi Q, Meyers LP, et al. on behalf of the Analysis and Research in Cancers of the Digestive System Group. Prognosis of patients with peritoneal metastatic colorectal cancer given systemic therapy: an analysis of individual patient data from prospective randomised trials from the Analysis and Research in Cancers of the Digestive System (ARCAD) database. Lancet Oncol 2016;17:1709-19.

14. Quenet F, Elias D, Roca L, et al. A UNICANCER phase III trial of hyperthermic intra-peritoneal chemotherapy (HIPEC) for colorectal peritoneal carcinomatosis. PRODIGE 7. Eur J Surg Oncol 2019;45:e17.

15. Dube P, Sideris L, Law C, et al. Guidelines on the use of cytoreductive surgery and hyperthermic intraperitoneal chemotherapy in patients with peritoneal surface malignancy arising from colorectal or appendiceal neoplasms. Curr Oncol 2015;22:e100-12.

16. Abboud K, Andre T, Brunel M, et al. Management of colorectal peritoneal metastases: expert opinion. J Visc Surg 2019;156:377-9.

17. Sanchez-Hidalgo JM, Rodriguez-Ortiz L, Arjona-Sanchez A, et al. Colorectal peritoneal metastases: optimal management review. World J Gastroenterol 2019;25:3484-502.

18. Chicago Consensus Working Group. The Chicago consensus on peritoneal surface malignancies: management of colorectal metastases. Ann Surg Oncol 2020;27:1761-7.

19. Klaver CEL, Groenen H, Morton DG, et al. Recommendations and consensus on the treatment of peritoneal metastases of colorectal origin: a systematic review of national and international guidelines. Colorectal Dis 2017;19:224-36.

20. Sugarbaker PH. Successful management of microscopic residual disease in large bowel cancer. Cancer Chemother Pharmacol 1999;43(suppl):S15-25.

21. Hribaschek A, Pross M, Kuhn R, et al. Prevention and treatment of peritoneal carcinomatosis in experimental investigations with CPT-11 and oxaliplatin. Anticancer Drugs 2002;13:605-14.

22. Naffouje SA, Salti GI. Cytoreductive surgery and hyperthermic intraperitoneal chemotherapy in elderly patients: complete cytoreduction is feasible and crucial for improved survival despite high carcinomatosis index. Anticancer Res 2018;38:441-8.

23. Wong EYT, Tan GHC, Chia CSL, Kumar M, Soo KC, Teo MCC. Morbidity and mortality of elderly patients following cytoreductive surgery and hyperthermic intraperitoneal chemotherapy (HIPEC). Asia PacJ Clin Oncol 2018;14:e193-202.

24. Cigdem Arslan N, Bisgin NT, Altay C, et al. Is advanced age a hesitation for cytoreductive surgery and hyperthermic intraperitoneal chemotherapy in colorectal cancer? J Buon 2018;23:77-83.

25. Gagnière J, Veziant J, Pereira B, et al. Cytoreductive surgery and hyperthermic intraperitoneal chemotherapy for the elderly: is it reasonable? A meta-analysis. Ann Surg Oncol 2018;25:709-19.

26. Lopez-Lopez V, Cascales-Campos PA, Schneider MA, et al. Cytoreductive surgery and hyperthermic intraperitoneal chemotherapy (HIPEC) in elderly patients. A systematic literature review. Surg Oncol 2016;25:378-84.

27. McPartland SJ, Goodman MD. The effect of elevated body mass index on outcomes following cytoreductive surgery with hyperthermic intraperitoneal chemotherapy. Ann Surg Oncol 2014;21:463-7.

28. Votanopoulos KI, Swords DS, Swett KR, et al. Obesity and peritoneal surface disease: outcomes after cytoreductive surgery with hyperthermic intraperitoneal chemotherapy for appendiceal and colon primary tumors. Ann Surg Oncol 2013;20:3899-904.

29. Naffouje SA, Tulla KA, Khalaf Z, Salti GI. The impact of вмI extremes on disease-free survival and overall survival following cytoreductive surgery and hyperthermic intraperitoneal chemotherapy. J Gastrointest Oncol 2019;10:194-202. 
30. de Bree E, Koops W, Kroger R, van Ruth S, Verwaal VJ, Zoetmulder FAN. Preoperative computed tomography and selection of patients with colorectal peritoneal carcinomatosis for cytoreductive surgery and hyperthermic intraperitoneal chemotherapy. Eur J Surg Oncol 2006;32:65-71.

31. Dromain C, Bisdorff A, Elias D, et al. Computed tomographic features of peritoneal carcinomatosis treated by intraperitoneal chemohyperthermia. J Comput Assist Tomogr 2003; 27:327-32.

32. Dromain C, Leboulleux S, Auperin A, et al. Staging of peritoneal carcinomatosis: enhanced CT vs. РЕT/СT. Abdom Imaging 2008;33:87-93.

33. Dresen RC, De Vuysere S, De Keyzer F, et al. Whole-body diffusion-weighted MRI for operability assessment in patients with colorectal cancer and peritoneal metastases. Cancer Imaging 2019;19:1.

34. van 't Sant I, van Eden WJ, Engbersen MP, et al. Diffusionweighted MRI assessment of the peritoneal cancer index before cytoreductive surgery. Br J Surg 2019;106:491-8.

35. Sasaki Y, Hamaguchi T, Yamada Y, et al. Value of $K R A S, B R A F$, and PIK3CA mutations and survival benefit from systemic chemotherapy in colorectal peritoneal carcinomatosis. Asian Pac J Cancer Prev 2016;17:539-43.

36. Liberale G, Van Den Eynde M, Hendlisz A, Nakadi EI. Recommendations for general surgeons facing incidental peritoneal carcinomatosis of colorectal origin. Eur J Surg Oncol 2008;34:725-6.

37. Swellengrebel HAM, Zoetmulder FAN, Smeenk RM, Antonini $\mathrm{N}$, Verwaal VJ. Quantitative intra-operative assessment of peritoneal carcinomatosis-a comparison of three prognostic tools. Eur J Surg Oncol 2009;35:1078-84.

38. Mercier F, Mohamed F, Cazauran JB, et al. An update of peritonectomy procedures used in cytoreductive surgery for peritoneal malignancy. Int J Hyperthermia 2019;36:744-52.

39. Jacquet P, Sugarbaker PH. Clinical research methodologies in diagnosis and staging of patients with peritoneal carcinomatosis. Cancer Treat Res 1996;82:359-74.

40. Maillet M, Glehen O, Lambert J, et al. on behalf of the BIGRENAPE Working Group. Early postoperative chemotherapy after complete cytoreduction and hyperthermic intraperitoneal chemotherapy for isolated peritoneal carcinomatosis of colon cancer: a multicenter study. Ann Surg Oncol 2016;23:863-9.

41. von Breitenbuch P, Boerner PT, Jeiter T, Piso P, Schlitt HJ. Laparoscopy as a useful selection tool for patients with prior surgery and peritoneal metastases suitable for multimodality treatment strategies. Surg Endosc 2018;32:2288-94.

42. Chua TC, Morris DL, Esquivel J. Impact of the peritoneal surface disease severity score on survival in patients with colorectal cancer peritoneal carcinomatosis undergoing complete cytoreduction and hyperthermic intraperitoneal chemotherapy. Ann Surg Oncol 2010;17:1330-6.

43. Piso P, Arnold D. Multimodal treatment approaches for peritoneal carcinosis in colorectal cancer. Dtsch Arztebl Int 2011; 108:802-8.

44. Elias D, Gilly F, Boutitie F, et al. Peritoneal colorectal carcinomatosis treated with surgery and perioperative intraperitoneal chemotherapy: retrospective analysis of 523 patients from a multicentric French study. J Clin Oncol 2010;28:63-8.

45. Winer J, Zenat $\mathrm{M}$, Ramalingam $\mathrm{L}$, et al. Impact of aggressive histology and location of primary tumor on the efficacy of surgical therapy for peritoneal carcinomatosis of colorectal origin. Ann Surg Oncol 2014;21:1456-62.

46. Yonemura Y, Canbay E, Ishibashi H. Prognostic factors of peritoneal metastases from colorectal cancer following cytoreductive surgery and perioperative chemotherapy. ScientificWorldJournal 2013;2013:978394.

47. Massalou D, Benizri E, Chevallier A, et al. Peritoneal carcinomatosis of colorectal cancer: novel clinical and molecular outcomes. Am J Surg 2017;213:377-87.

48. Esquivel J, Sticca R, Sugarbaker P, et al. Cytoreductive surgery and hyperthermic intraperitoneal chemotherapy in the management of peritoneal surface malignancies of colonic origin: a consensus statement. Society of Surgical Oncology. Ann Surg Oncol 2007;14:128-33.

49. Sugarbaker PH. Cytoreductive surgery using peritonectomy and visceral resections for peritoneal surface malignancy. Transl Gastrointest Cancer 2013;2:54-74.

50. Spiliotis J, Kalles V, Kyriazanos I, et al. CRS and HIPEC in patients with peritoneal metastasis secondary to colorectal cancer: the small-bowel PCI score as a predictor of survival. Pleura Peritoneum 2019;4:20190018.

51. Jacoby H, Berger Y, Barda L, et al. Implications of stoma formation as part of cytoreductive surgery and hyperthermic intraperitoneal chemotherapy. World J Surg 2018;42:2036-42.

52. Stiles ZE, Hinkle NM, Munene G, et al. The impact of ostomy creation after cytoreduction and hyperthermic intraperitoneal chemotherapy in a newly established peritoneal malignancy program. Am Surg 2018;84:776-82.

53. Doud AN, Levine EA, Fino NF, Stewart JH, Shen P, Votanopoulos KI. Stoma creation and reversal after cytoreductive surgery with hyperthermic intraperitoneal chemotherapy. Ann Surg Oncol 2016;23:503-10.

54. von Breitenbuch P, Piso P, Schlitt HJ. Safety of rectum anastomosis after cytoreductive surgery and hyperthermic intraperitoneal chemotherapy. J Surg Oncol 2018;118:551-6.

55. Glehen O, Cotte E, Kusamura S, et al. Hyperthermic intraperitoneal chemotherapy: nomenclature and modalities of perfusion. J Surg Oncol 2008;98:242-6.

56. Elias D, Lefevre JH, Chevalier J, et al. Complete cytoreductive surgery plus intraperitoneal chemohyperthermia with oxaliplatin for peritoneal carcinomatosis of colorectal origin. JClin Oncol 2009;27:681-5.

57. Elias D, Benizri E, Di Pietrantonio D, Menegon P, Malka D, Raynard B. Comparison of two kinds of intraperitoneal chemotherapy following complete cytoreductive surgery of colorectal peritoneal carcinomatosis. Ann Surg Oncol 2007;14:509-14.

58. Lemoine L, Sugarbaker P, Van der Speeten K. Drugs, doses, and durations of intraperitoneal chemotherapy: standardising HIPEC and EPIC for colorectal, appendiceal, gastric, ovarian peritoneal surface malignancies and peritoneal mesothelioma. Int J Hyperthermia 2017;33:582-92.

59. Wisselink DD, Braakhuis LLF, Gallo G, et al. Systematic review of published literature on oxaliplatin and mitomycin $\mathrm{C}$ as chemotherapeutic agents for hyperthermic intraperitoneal chemotherapy in patients with peritoneal metastases from colorectal cancer. Crit Rev Oncol Hematol 2019;142:119-29.

60. Tan GHC, Shannon NB, Chia CS, Soo KC, Teo MCC. Platinum agents and mitomycin C-specific complications in cytoreductive surgery (CRS) and hyperthermic intraperitoneal chemotherapy (HIPEC). Int J Hyperthermia 2018;34:595-600.

61. Hompes D, D'Hoore A, Wolthuis A, et al. The use of oxaliplatin or mitomycin C in HIPEC treatment for peritoneal carcinomatosis from colorectal cancer: a comparative study. J Surg Oncol 2014;109:527-32.

62. Narasimhan V, Warrier S, Michael M, Ramsay R, Heriot A. Oxaliplatin versus mitomycin $\mathrm{C}$ following complete cytoreduction for colorectal peritoneal metastases: a comparative study. J Gastrointest Surg 2020;24:2104-12.

63. Lam JY, McConnell YJ, Rivard JD, Temple WJ, Mack LA. Hyperthermic intraperitoneal chemotherapy + early postoperative intraperitoneal chemotherapy versus hyperthermic intraperitoneal chemotherapy alone: assessment of survival outcomes for colorectal and high-grade appendiceal peritoneal carcinomatosis. Am J Surg 2015;210:424-30. 
64. Tan GH, Ong WS, Chia CS, Tham CK, Soo KC, Teo MC. Does early post-operative intraperitoneal chemotherapy (EPIC) for patients treated with cytoreductive surgery and hyperthermic intraperitoneal chemotherapy (HIPEC) make a difference? Int J Hyperthermia 2016;32:281-8.

65. Huang Y, Alzahrani NA, Liauw W, Soudy H, Alzahrani AM, Morris DL. Early postoperative intraperitoneal chemotherapy is associated with survival benefit for appendiceal adenocarcinoma with peritoneal dissemination. Eur J Surg Oncol 2017;43:2292-8.

66. Huang Y, Alzahrani NA, Liauw W, Traiki TB, Morris DL. Early postoperative intraperitoneal chemotherapy for low-grade appendiceal mucinous neoplasms with pseudomyxoma peritonei: is it beneficial? Ann Surg Oncol 2017;24:176-83.

67. Soucisse ML, Liauw W, Hicks G, Morris DL. Early postoperative intraperitoneal chemotherapy for lower gastrointestinal neoplasms with peritoneal metastasis: a systematic review and critical analysis. Pleura Peritoneum 2019;4:20190007.

68. Barrios Sanchez P. Radical treatment of peritoneal carcinomatosis: times are changing. Cir Esp 2019;97:125-7.

69. Smith ME, Nathan H. Cytoreductive surgery and hyperthermic intraperitoneal chemotherapy: safety is only half of the story. JAMA Netw Open 2019;2:e186839.

70. Liberale G, Ameye L, Hendlisz A. PRODIGE 7 should be interpreted with caution. Acta Chir Belg 2019;119:263-6.

71. Ceelen W. HIPEC with oxaliplatin for colorectal peritoneal metastasis: the end of the road? Eur J Surg Oncol 2019;45:400-2.

72. Auer RC, Sivajohanathan D, Biagi J, et al. Indications for hyperthermic intraperitoneal chemotherapy with cytoreductive surgery: a systematic review. Eur J Cancer 2020;127:76-95.

73. Burnett A, Lecompte MA, Trabulsi N, et al. Peritoneal carcinomatosis index predicts survival in colorectal patients undergoing HIPEC using oxaliplatin: a retrospective single-arm cohort study. World J Surg Oncol 2019;17:83.

74. Waite K, Youssef H. The role of neoadjuvant and adjuvant systemic chemotherapy with cytoreductive surgery and heated intraperitoneal chemotherapy for colorectal peritoneal metastases: a systematic review. Ann Surg Oncol 2017; 24:705-20.

75. Rovers KP, Bakkers C, Simkens G, et al. Perioperative systemic therapy and cytore ductive surgery with HIPEC versus upfront cytoreductive surgery with HIPEC alone for isolated resectable colorectal peritoneal metastases: protocol of a multicentre, open-label, parallel-group, phase II-III, randomised, superiority study (CAIRO6). BMC Cancer 2019;19:390.

76. Devilee RA, Simkens GA, van Oudheusden TR, et al. Increased survival of patients with synchronous colorectal peritoneal metastases receiving preoperative chemotherapy before cytoreductive surgery and hyperthermic intraperitoneal chemotherapy. Ann Surg Oncol 2016;23:2841-8.

77. Leimkuhler M, Hemmer PHJ, Reyners AKL, et al. Neoadjuvant chemotherapy followed by cytoreductive surgery and hyperthermic intraperitoneal chemotherapy for colorectal cancer: a feasibility and safety study. World J Surg Oncol 2019;17:14.

78. Ceelen W, Van Nieuwenhove Y, Putte DV, Pattyn P. Neoadjuvant chemotherapy with bevacizumab may improve outcome after cytoreduction and hyperthermic intraperitoneal chemoperfusion (HIPEC) for colorectal carcinomatosis. Ann Surg Oncol 2014;21:3023-8.

79. Palmieri LJ, Fihri A, Doat S, et al. Tumor-size responses to firstline is a predictor of overall survival in metastatic colorectal cancer. Eur Radiol 2019;29:3871-80.

80. Cassidy J, Clarke S, Díaz-Rubio E, et al. Randomized phase III study of capecitabine plus oxaliplatin compared with fluorouracil/folinic acid plus oxaliplatin as first-line therapy for metastatic colorectal cancer. J Clin Oncol 2008;26:2006-12.

81. Guo Y, Xiong BH, Zhang T, Cheng Y, Ma L. XELOX vs. FOLFOX in metastatic colorectal cancer: an updated meta-analysis. Cancer Invest 2016;34:94-104.

82. Al-Hajeili M, Marshall JL, Smaglo BG. Neoadjuvant treatment for surgically resectable metastatic colorectal cancer. Oncology (Williston Park) 2016;30:10-16.

83. Yamazaki K, Nagase M, Tamagawa H, et al. Randomized phase III study of bevacizumab plus FOLFIRI and bevacizumab plus mFOLFOX6 as first-line treatment for patients with metastatic colorectal cancer (WJOG4407G). Ann Oncol 2016;27:1539-46.

84. Neugut AI, Lin A, Raab GT, et al. FOLFOX and FOLFIRI use in stage IV colon cancer: analysis of SEER-Medicare data. Clin Colorectal Cancer 2019;18:133-40.

85. Eveno C, Passot G, Goere D, et al. Bevacizumab doubles the early postoperative complication rate after cytoreductive surgery with hyperthermic intraperitoneal chemotherapy (HIPEC) for peritoneal carcinomatosis of colorectal origin. Ann Surg Oncol 2014;21:1792-800.

86. Rosen LS, Jacobs IA, Burkes RL. Bevacizumab in colorectal cancer: current role in treatment and the potential of biosimilars. Target Oncol 2017;12:599-610.

87. Wrobel P,Ahmed S. Current status of immunotherapy in metastatic colorectal cancer. Int J Colorectal Dis 2019;34:13-25.

88. Ciliberto D, Staropoli N, Caglioti F, et al. The best strategy for $R A S$ wild-type metastatic colorectal cancer patients in first-line treatment: a classic and Bayesian meta-analysis. Crit Rev Oncol Hematol 2018;125:69-77.

89. Bridgewater JA, Pugh SA, Maishman T, et al. on behalf of the NewEPOC investigators. Systemic chemotherapy with or without cetuximab in patients with resectable colorectalliver metastasis (New EPOC): long-term results of a multicentre, randomised, controlled, phase 3 trial. Lancet Oncol 2020;21:398-411.

90. Wong JSM, Tan GHC, Chia CS, Ong J, Ng WY, Teo MCC. The importance of synchronicity in the management of colorectal peritoneal metastases with cytoreductive surgery and hyperthermic intraperitoneal chemotherapy. World J Surg Oncol 2020;18:10.

91. Braam HJ, van Oudheusden TR, de Hingh IH, et al. Patterns of recurrence following complete cytoreductive surgery and hyperthermic intraperitoneal chemotherapy in patients with peritoneal carcinomatosis of colorectal cancer. J Surg Oncol 2014;109:841-7.

92. Hentzen JERK, Rovers KP, Kuipers H, et al. Impact of synchronous versus metachronous onset of colorectal peritoneal metastases on survival outcomes after cytoreductive surgery (CRS) with hyperthermic intraperitoneal chemotherapy (HIPEC): a multicenter, retrospective, observational study. Ann Surg Oncol 2019;26:2210-21.

93. Choudry HA, Bednar F, Shuai Y, et al. Repeat cytoreductive surgery-hyperthermic intraperitoneal chemoperfusion is feasible and offers survival benefit in select patients with peritoneal metastases. Ann Surg Oncol 2019;26:1445-53.

94. Jost E, Mack LA, Sideris L, et al. Evaluation of repeat cytoreductive surgery and heated intraperitoneal chemotherapy for patients with recurrent peritoneal carcinomatosis from appendiceal and colorectal cancers: a multicentre Canadian study. Can J Surg 2020;63:E71-9.

95. Kwakman R, Schrama AM, van Olmen JP, et al. Clinicopathological parameters in patient selection for cytoreductive surgery and hyperthermic intraperitoneal chemotherapy for colorectal cancer metastases: a meta-analysis. Ann Surg 2016;263:1102-11.

96. Lorimier G, Linot B, Paillocher N, et al. Curative cytoreductive surgery followed by hyperthermic intraperitoneal chemotherapy in patients with peritoneal carcinomatosis and synchronous resectable liver metastases arising from colorectal cancer. Eur J Surg Oncol 2017;43:150-8.

97. Gelli M, Huguenin JFL, de Baere T, et al. Peritoneal and 
extraperitoneal relapse after previous curative treatment of peritoneal metastases from colorectal cancer: what survival can we expect? Eur J Cancer 2018;100:94-103.

98. El-NakeepS, Rashad N, Oweira H, etal. Intraperitoneal chemotherapy and cytoreductive surgery for peritoneal metastases coupled with curative treatment of colorectal liver metastases: an updated systematic review. Expert Rev Gastroenterol Hepatol 2017;11:249-58.

99. Baratti D, Kusamura S, Iusco D, et al. Should a history of extraperitoneal disease be a contraindication to cytoreductive surgery and hyperthermic intraperitoneal chemotherapy for colorectal cancer peritoneal metastases? Dis Colon Rectum 2018;61:1026-34.

100. Pinto A, Hobeika C, Philis A, Kirzin S, Carrère N, Ghouti L. Synchronous liver metastases and peritoneal carcinomatosis from colorectal cancer: different strategies for curative treatment? Langenbecks Arch Surg 2019;404:477-88.

101. Elias D, Benizri E, Pocard M, et al. Treatment of synchronous peritoneal carcinomatosis and liver metastases from colorectal cancer. Eur J Surg Oncol 2006;32:632-6.

102. Bushati M, Rovers KP, Sommariva A, et al. The current practice of cytoreductive surgery and HIPEC for colorectal peritoneal metastases: results of a worldwide Web-based survey of the Peritoneal Surface Oncology Group International (PSOGI). Eur J Surg Oncol 2018;44:1942-8.

103. van der Werf LR, Wassenaar E, de Niet A, et al. The impact of radiological retroperitoneal lymphadenopathy on survival after cytoreductive surgery and hyperthermic intraperitoneal chemotherapy for colorectal peritoneal metastases. Eur J Surg Oncol 2019;45:376-82.
104. Elias D, Honore C, Dumont F, et al. Results of systematic second-look surgery plus HIPEC in asymptomatic patients presenting a high risk of developing colorectal peritoneal carcinomatosis. Ann Surg 2011;254:289-93.

105. Goere D, Glehen O, Quenet F, et al. Results of a randomized phase 3 study evaluating the potential benefit of a second-look surgery plus HIPEC in patients at high risk of developing colorectal peritoneal metastases (PROPHYLOCHIP-NTC01226394) [abstract 3531]. J Clin Oncol 2018;36:. [Available online at: https://ascopubs.org/doi/abs/10.1200/JCO.2018.36.15_suppl. 3531; cited 16 November 2020]

106. Klaver CEL, Wisselink DD, Punt CJA, et al. Adjuvant HIPEC in patients with colon cancer at high risk of peritoneal metastases: primary outcome of the COLOPEC multicenter randomized trial [abstract 482]. JClin Oncol 2019;37:. [Available online at: https://ascopubs.org/doi/abs/10.1200/JCO.2019.37.4_suppl. 482; cited 16 November 2020]

107. Bastiaenen VP, Klaver CEL, Kok NFM, et al. Second and third look laparoscopy in pT4 colon cancer patients for early detection of peritoneal metastases; the COLOPEC 2 randomized multicentre trial. BMC Cancer 2019;19:254.

108. Kurtz F, Struller F, Horvath P, et al. Feasibility, safety, and efficacy of pressurized intraperitoneal aerosol chemotherapy (PIPAC) for peritoneal metastasis: a registry study. Gastroenterol Res Pract 2018;2018:2743985.

109. Alyami M, Mercier F, Siebert M, etal. Unresectable peritoneal metastasis treated by pressurized intraperitoneal aerosol chemotherapy (PIPAC) leading to cytoreductive surgery and hyperthermic intraperitoneal chemotherapy. EurJSurg Oncol 2019;:[online ahead of print]. 